\title{
Physiologically based control laws featuring antagonistic muscle co-activation for stable compliant joint drives
}

\author{
Salvatore Annunziata* and Axel Schneider \\ Research Group Mechatronics of Biomimetic Actuators, Faculty of Technology, University of Bielefeld, \\ Bielefeld, Germany
}

\begin{abstract}
The development of robot joints ensuring controllable compliant behaviors during interaction tasks has gained the interest of the robot community in the recent years. In biological systems, while intrinsic muscle nonlinear elasticity allows the regulation of joint compliance through antagonistic co-activation, physiological control mechanisms ensure robust stability during interaction tasks both in static cases and during motion. This work presents a novel control approach for the simultaneous regulation of position and stiffness in a hinge joint driven by two muscles which combines biological findings like co-activation and reproduces reflex actions and additional influences to ensure stability. Based on the analysis of the stiffness generated in the antagonistic system and on the evaluation of stability issues when the musculoskeletal setup is loaded by external forces, a stiffness controller is proposed which integrates a stiffness computation block and an adaptive mechanism for the control of stability during interactions. The position controller, modeling physiological properties and motor-neurons, relies on reciprocal activation while the stiffness controller implements a co-activation strategy. The controller is tested in a numerical simulation using Matlab/Simulink ${ }^{\circledR}$ for different task conditions. Simulation results demonstrate the ability of the controller to simultaneously regulate position and adapt joint compliance to different external perturbations.
\end{abstract}

Keywords: Antagonistic joints, bio-inspired control systems, biological system modeling, co-activation, compliance control

\section{Introduction}

Modern robotic research focuses the attention on the improvement of safety during contacts with humans. The desire to reduce risks when a human-machine interaction takes place could be fulfilled with the construction of elastic and lightweight systems. The introduction of elasticity in the joints and the control of joint compliance are considered to be the key elements for safe interaction [1, 2]. Biological systems are elastic and have the ability to regulate joint

*Corresponding author: Salvatore Annunziata, Faculty of Technology, Mechatronics of Biomimetic Actuators, University of Bielefeld, PO Box 1001 31, 33501 Bielefeld, Germany. Tel: +49 5211065172; Fax: +49 521106440; E-mail: salvatore.annunziata@uni-bielefeld.de. compliance through antagonistic muscle co-activation due to the intrinsic muscle nonlinear elasticity [3]. Research in biomechanics has suggested that coactivation and movement of a limb can be controlled separately. According to Yamazaki and co-workers $[4,5]$ humans can modulate reciprocal activity and co-activation independently. Other studies suggest that joint stiffness and movements are closely connected $[6,7]$. Co-activation of antagonistic muscles affects also stability of the limb both in static cases and during movements $[7,8]$. The musculoskeletal system is a highly nonlinear elastic actuation system in which the muscle, with its intrinsic viscoelastic properties, is the key actuator. The central nervous system (CNS) is responsible for controlling the movement and the necessary compliance at the same time for achieving 
robust stability during interaction tasks. Therefore, it seems to be advisable to observe physiological control mechanisms based on nonlinear muscle models during motion to implement successful solutions for the construction and control of compliant robot joints.

An early attempt to apply knowledge of human physiological motion control in robotics is found in [9] in which antagonistic pneumatic actuators pulling on an elbow joint setup are controlled by artificial neural networks based on physiological laws. In the last years many studies have been focusing on the implementation of impedance control strategies based on learning processes and optimization algorithms [10-13]. Burdet et al. [10] and Hoult, Cole [11] studied a neuromuscular model in which feed-forward estimate of counteracting external forces were based on internal models. In his inspiring work [12] Franklin introduced an algorithm of motor learning, deduced from observations in psychophysical experiments. The motor commands are iteratively adjusted based on a V-shaped learning function. The process makes use of feedback and feedforward signals. In [13] the idea to control antagonistic joint impedance in reaching tasks originated from the optimization of a cost function based on the position error and the formalization of internal models for minimization of energy consumption. In those works, muscles are typically modeled as linear spring-damper systems in which stiffness and damping increase with activation.

In this work we develop a novel impedance control approach for stable compliant actuation which takes advantage of nonlinear muscle models in an antagonistic arrangement, together with adaptive regulation and modeling of physiological properties. In particular, virtual antagonistic actuation systems based on Hill-type muscle models $[14,15]$ together with physiologically based control laws featuring co-activation and adaptive regulation of joint compliance are proposed to simultaneously control position and stiffness. In addition, aspects of system stability are integrated into the system. Two antagonistic muscles drive a hinge joint and receive the control signals (activations) from a position controller and a stiffness controller. A stiffness computation block calculates the actual stiffness based on inherent nonlinear properties and actual activations of the muscles [16]. The stiffness controller is a PI-type and acts on all antagonistic muscle thus implementing co-activation. An additional adaptive control mechanism modifies the stiffness set-point to ensure stability when external perturbations are applied to the joint. The position controller acts on one muscle at a time and combines a feedback loop with feed-forward commands reproducing reflex actions and additional biological influences.

The work is structured as follows. In section 2 , the model of a hinge joint moved by two muscles is introduced and the mathematical description of the muscle model is given. In section 3 , the joint stiffness for the given antagonistic setup is analyzed and an expression for its computation is proposed. In section 4 , a stability analysis is performed and simulation results are discussed when muscles are controlled with increasing activations. Section 5 describes the bioinspired position and stiffness control schemes. The controller is tested in a numerical simulation using Matlab/Simulink ${ }^{\circledR}$ for different task conditions (section 6). Simulation results confirm the ability of the control scheme to simultaneously regulate the position and, with respect to different external perturbations, adapt the joint compliance. Our results are in line with the results proposed in literature $[7,17]$.

\section{Biological system modeling}

This study concentrates on the concurrent control of position and stiffness of a joint driven by two muscles. The biomechanical system adopted in this work is a one-degree-of-freedom (DoF) rotational hinge joint constrained to move in the horizontal plane. An extremely simplified human arm with only two muscles pulling upon the elbow joint will be used as biological reference in this manuscript (Fig. 1a). In order to characterize joint stiffness and to test the concurrent control of position and stiffness, a simplified model of the biological system has to be defined. A simple hinge joint geometry with a pulley is assumed for the arm. The muscles are modeled based on a Hill-type model [14]. For the bio-inspired joint model, several physiological parameters have to be selected. In this work, the parameters for both arm model and muscle model are obtained either directly from literature or selected in order to match empirical data (as in the case of joint stiffness and viscosity).

\subsection{Arm model}

Figure 1a shows a simplified version of the human arm with the elbow joint moved by two muscles ( $m u s$ cle 1 is the extensor and muscle 2 is the flexor). The 


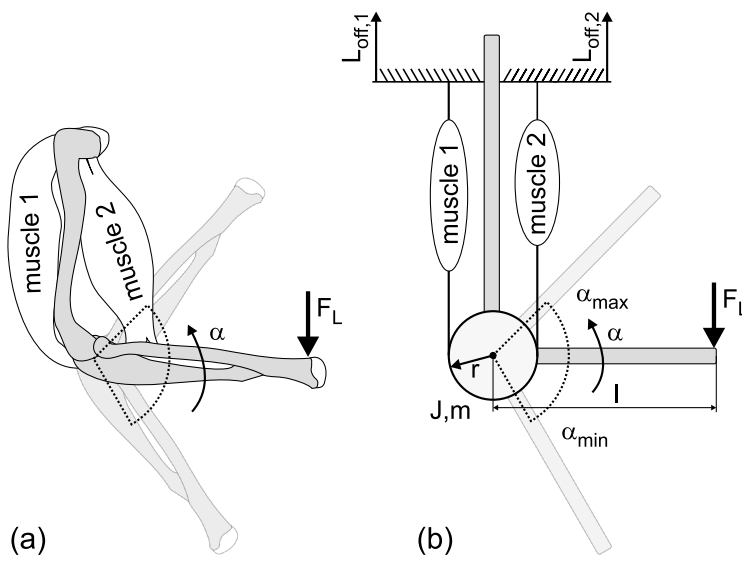

Fig. 1. (a) Simplified depiction of the human arm with an elbow joint moved by two muscles. (b) Model of the antagonistic joint setup in (a).

joint angle $\alpha$ is defined as $\alpha_{0}=0$ when the forearm is in the horizontal position and the angular joint range is limited to:

$$
\alpha_{\min } \leq \alpha(t) \leq \alpha_{\max }
$$

with $\alpha_{\min }=-\frac{\pi}{3}$ rad and $\alpha_{\max }=\frac{\pi}{4}$ rad. The working range is depicted with a dashed arc. A simplified model of the human forearm can be described by a 1-link manipulator with a hinge joint moved by two muscles. In order to compute the length and the moment arms for the muscles as a function of the joint angle, a model of the joint has to be chosen. In general, the moment arm for each muscle in the elbow joint is a nonlinear function of the joint angle. However, in this paper, a constant moment arm is assumed. The use of constant moment arms is a simplification that allows a closed mathematical description of stiffness and is consistent with the assumptions made by, e.g., the models of Gribble [6]. For parameter fitting of muscle data it can be assumed that this assumption degrades by $30 \%$ over a movement range of $1.66 \mathrm{rad}$ [18]. This is however not focused on in the present work. Figure $1 \mathrm{~b}$ shows the model of the antagonistic setup depicted in (a) as it will be used for the analysis of stiffness and for the concurrent control of position and stiffness. $L_{\text {off }, 1}$ and $L_{\text {off, } 2}$ represent pre-stretch lengths of the muscles when integrated into the mechanical joint setup. The center of gravity of the forearm is assumed to be at the center of the lever. $m$ is the mass of the lever arm concentrated in the center of gravity and $l$ is the distance from the end of the lever to the axis of rotation of the
Table 1

Joint parameters for the antagonistic setup ([19-21])

\begin{tabular}{llcc}
\hline Variable & Description & Value & Unit \\
\hline$r$ & Pulley radius & 0.03 & {$[\mathrm{~m}]$} \\
$\mathrm{J}$ & Moment of inertia & 0.06125 & {$\left[\mathrm{~kg} \mathrm{~m}^{2}\right]$} \\
$\mathrm{m}$ & Mass lever arm & 1.5 & {$[\mathrm{~kg}]$} \\
1 & Length lever arm & 0.35 & {$[\mathrm{~m}]$} \\
$\mathrm{K}_{\max }$ & Max joint stiffness & $35^{1}$ & {$[\mathrm{Nm} / \mathrm{rad}]$} \\
$\mathrm{B}_{\max }$ & Max joint viscosity & $4^{1}$ & {$[\mathrm{Nms} / \mathrm{rad}]$} \\
${ }^{1}$ For max co-activation and low velocity. & &
\end{tabular}

pulley. $J$ is the moment of inertia of the forearm and $r$ is the radius of the pulley.

The physical parameters of the described setup have been obtained from [19-21] and are reported in Table 1 (where the length of the moment arm corresponds to the average value of anatomical data). The calculation of the moment of inertia of the forearm is given in the Appendix A.

When the lever arm moves, each muscle length differs from the resting length $L_{0}$ by an effective deviation given by

$$
\begin{aligned}
& \Delta L_{\text {eff }, 1}=\Delta L_{1}+L_{\text {off }, 1} \text { with } \Delta L_{1}=r \cdot \alpha \\
& \Delta L_{\text {eff }, 2}=\Delta L_{2}+L_{\text {off }, 2} \text { with } \Delta L_{2}=-r \cdot \alpha .
\end{aligned}
$$

\subsection{Muscle model}

The force generated by each muscle is obtained by using a Hill-type muscle model [14]. In this model, the force produced by a muscle depends on the muscle activation, and varies with muscle length $L$ and with the shortening/lengthening velocity $V$ of the muscle. Moreover, muscle force-length dependency is divided in an active and a passive component. Therefore, the overall time varying muscle force $F_{M}$ can be mathematically described as

$$
F_{M}=\left[F_{L a}(L) \cdot F_{V}(V) \cdot a(t)+F_{L p}(L)\right] \cdot F_{\max }
$$

where $F_{L a}(L)$ is the active length-dependent force component, $F_{V}(V)$ is the velocity-dependent force component and $F_{L p}(L)$ is the passive length-dependent force component. All functions are normalized to maximum isometric muscle force $F_{\max }$, optimal muscle length $L_{0}$ (length at which the active muscle force reaches its maximum) and maximum shortening velocity $V_{\max } \cdot a(t)$ is the normalized time varying muscle activation. The analytical expressions of the functions are approximations of the dimensionless curves shown by Zajac [15]. 
The active force component is assumed to be a quadratic function of the length

$$
F_{L a}\left(\Delta L_{\mathrm{eff}}\right)= \begin{cases}1-\left(\frac{\Delta L_{\mathrm{eff}}}{w_{L 0}}\right)^{2} & \left|\Delta L_{\mathrm{eff}}\right|<w_{L 0} \\ 0 & \text { otherwise }\end{cases}
$$

where $w_{L 0}$ corresponds to the product $w \cdot L_{0}$ in which $w$ is the half width of the active muscle curve. $\Delta L_{\mathrm{eff}}$ has been defined in (1).

The passive force component is described by the following equation

$$
F_{L p}\left(\Delta L_{\text {eff }}\right)=\left\{\begin{array}{lr}
K_{p} \cdot\left(\frac{\Delta L_{\text {eff }}}{p_{L 0}}\right)^{2} & \frac{\Delta L_{\text {eff }}}{p_{L 0}} \geq 0 \\
0 & \text { otherwise }
\end{array}\right.
$$

where the parameter $K_{p}$ changes the curvature of the curve and $p_{L 0}$ is a length scaling factor with respect to $L_{0}$.

The force-velocity dependency is approximated as

$$
F_{V}(V)= \begin{cases}\frac{V_{\max }-V}{V_{\max }+K_{v} \cdot V} & V>0 \\ N_{\mathrm{n}}+\left(N_{\mathrm{n}}-1\right) \cdot \frac{V_{\max }+V}{7.56 \cdot K_{v} \cdot V-V_{\max }} & V \leq 0\end{cases}
$$

Table 2

Muscle parameters hinge joint

\begin{tabular}{llccc}
\hline Variable & Description & $M_{1}$ & $M_{2}$ & Unit \\
\hline$L_{\text {off }}$ & Pre-stretch & 0.0 & -0.035 & {$[\mathrm{~m}]$} \\
$w_{L 0}$ & Half width F-L curve-opt. length & 0.08 & 0.06 & {$[\mathrm{~m}]$} \\
$F_{\max }$ & Max. isometric force & 800 & 1200 & {$[\mathrm{~N}]$} \\
$K_{\mathrm{p}}$ & Passive curve const. & 2 & 2 & \\
$p_{L 0}$ & Length scaling factor & 0.14 & 0.08 & {$[\mathrm{~m}]$} \\
$V_{\max }$ & Max short. velocity & 150 & 150 & {$[\mathrm{~m} / \mathrm{s}]$} \\
$N_{\mathrm{n}}$ & Max $F / F_{\max }$ at $-V_{\max }$ & 1.8 & 1.8 & \\
$K_{\mathrm{V}}$ & Curvature constant F-V curve & 5 & 5 & \\
\hline
\end{tabular}

the values of elbow joint stiffness and viscosity, experimentally measured in $[20,21]$ and reported in Table 1. The selected parameters for muscle 1 ( $M_{1}$, extensor muscle) and muscle 2 ( $M_{2}$, flexor muscle) are supplied in Table 2.

The forces generated by the antagonistic muscles in the model can be depicted with respect to the angle of joint rotation $\alpha$. Because of the joint setup, the muscles are coupled by the skeletal framework and therefore

heir force length curves are superimposed. Figure $2 \mathrm{a}$ depicts the force-angle relationships of muscle 1 and muscle 2 for the pulley joint when $\omega=0$ for maximum activations $\left(a_{1}=a_{2}=1\right)$. The black curve depicts the normalized force generated by muscle 1 , the gray curve shows the mirrored normalized force for muscle 2 .

\section{Joint stiffness}

The dynamic model equations of the system are:

$$
\begin{cases}\dot{\alpha} & =\omega \\ J \cdot \frac{\partial \omega}{\partial t} & =\tau-\tau_{G}-\tau_{L}\end{cases}
$$

where $\tau$ is the net torque of all antagonistic muscles pulling on the joint

$$
\tau=\tau_{2}-\tau_{1},
$$

$\tau_{L}$ is the load torque

$$
\tau_{L}=F_{L} \cdot l
$$

and $\tau_{G}$ is the gravitational torque about the joint

$$
\tau_{G}=m \cdot g \cdot \frac{l}{2} \cdot \cos (\alpha)
$$
vature constant in the $F_{V}$ curve are selected based on 
where $g$ is the acceleration due to gravity. With the objective of realizing a simultaneous control of position and stiffness for the antagonistic setup described above, joint stiffness has to be characterized and analyzed. The CNS modulates joint stiffness by acting either on individual muscle stiffness, or simultaneously activating all antagonistic muscles working on a joint (co-activation) [23, 24]. Changing co-activation can increase stiffness 4 to 7 times more than single muscle contraction [25].

\subsection{Stiffness generated by two antagonistic muscles in a pulley joint}

The expression of the stiffness generated by the antagonistically actuated system described above, can be obtained by calculating the first derivative of the net joint torque $\tau$ with respect to the joint angle $\alpha$
It can be seen that - once the geometrical muscle parameters are fixed - the stiffness is a function of the joint angle, its derivative and the two activations [16]. Figure $2 b$ shows the joint stiffness plotted over the joint angle $\alpha$ in the case of equal levels of activation for both muscles (co-activation) and $\omega=0$. As joint velocity is assumed to be zero, only forcelength curves are responsible for stiffness changes. The force-length curve is a quadratic approximation and as such causes a discontinuity at $\alpha \approx 0.8 \mathrm{rad}$ corresponding to the shortest muscle length in $M_{2}$ for which active force is generated [cf. Fig. 2a]. During movement, joint stiffness is subject to the force-velocity dependency of muscles which lead to the concepts of dynamic stiffness and viscosity. In [19] dynamic joint stiffness during movement is defined and the derivation of joint stiffness and viscosity is explained. In [26]

$$
\begin{aligned}
K= & -\frac{\partial \tau}{\partial \alpha}=-\frac{\partial\left(F_{M, 2} \cdot r-F_{M, 1} \cdot r\right)}{\partial \alpha} \\
= & -\frac{\partial\left[\left(F_{L a}\left(\Delta L_{\mathrm{eff}, 2}\right) \cdot F_{V}\left(V_{2}\right) \cdot a_{2}+F_{L p}\left(\Delta L_{\mathrm{eff}, 2}\right)\right) \cdot F_{\max , 2} \cdot r\right]}{\partial \alpha} \\
& +\frac{\partial\left[\left(F_{L a}\left(\Delta L_{\mathrm{eff}, 1}\right) \cdot F_{V}\left(V_{1}\right) \cdot a_{1}+F_{L p}\left(\Delta L_{\mathrm{eff}, 1}\right)\right) \cdot F_{\max , 1} \cdot r\right]}{\partial \alpha}
\end{aligned}
$$

and by inserting (1), (3), (4), (5) and (6) this yields

$$
\begin{aligned}
& K\left(\alpha, \omega, a_{1}, a_{2}\right)=2 \cdot r^{2} . \\
& \begin{cases}-\frac{F_{\max , 1} \cdot a_{1} \cdot\left(L_{\mathrm{off}, 1}+r \cdot \alpha\right)}{w_{L 01}^{2}} & \text { if } w_{L 01}>\left|L_{\mathrm{off}, 1}+r \cdot \alpha\right| \\
0 & \text { otherwise }\end{cases} \\
& + \begin{cases}\frac{r \cdot \omega+V_{\max }}{-K_{v} \cdot r \cdot \omega+V_{\max }} & \text { if } \omega<0 \\
N_{n}+\frac{\left(N_{n}-1\right) \cdot\left(V_{\max }-r \cdot \omega\right)}{-7.56 \cdot K_{v} \cdot r \cdot \omega-V_{\max }} & \text { otherwise }\end{cases} \\
& + \begin{cases}F_{\max , 1} \cdot K_{p, 1} \cdot \frac{\left(L_{\mathrm{off}, 1}+r \cdot \alpha\right)}{p_{L 0,1}^{2}} & \text { if } \alpha>-\frac{L_{\mathrm{off}, 1}}{r} \\
0 & \text { otherwise }\end{cases} \\
& - \begin{cases}\frac{F_{\max , 2} \cdot a_{2} \cdot\left(L_{\mathrm{off}, 2}-r \cdot \alpha\right)}{w_{L 02}^{2}} & \text { if } w_{L 02}>\left|L_{\mathrm{off}, 2}-r \cdot \alpha\right| \\
0 & \text { otherwise }\end{cases} \\
& +\left\{\begin{array}{ll}
\frac{-r \cdot \omega+V_{\max }}{K_{v} \cdot r \cdot \omega+V_{\max }} & \text { if } \omega>0 \\
N_{n}+\frac{\left(N_{n}-1\right) \cdot\left(V_{\max }+r \cdot \omega\right)}{7.56 \cdot K_{v} \cdot r \cdot \omega-V_{\max }} & \text { otherwise } \\
F_{\max , 2} \cdot K_{p, 2} \cdot \frac{\left(L_{\mathrm{off}, 2}-r \cdot \alpha\right)}{p_{L 0,2}^{2}} & \text { if } \alpha<\frac{L_{\mathrm{off}, 2}}{r} \\
0 & \text { otherwise }
\end{array}\right) .
\end{aligned}
$$



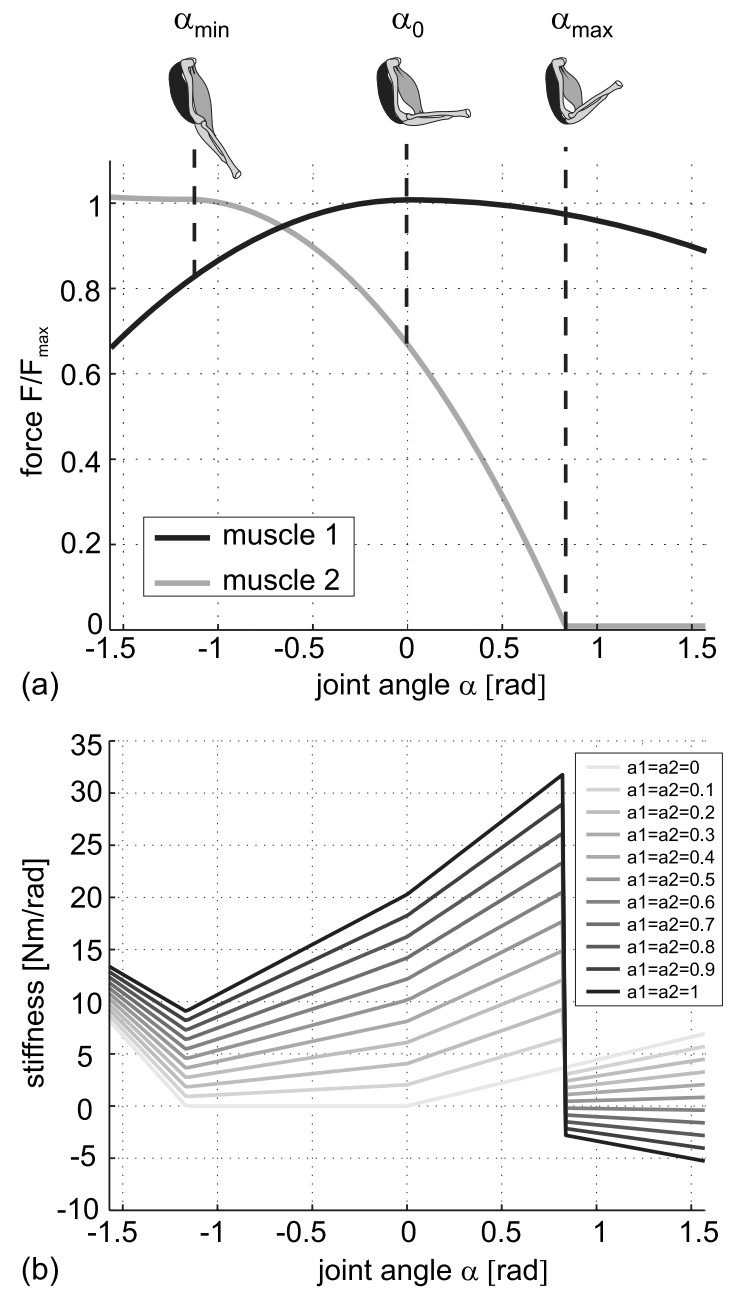

Fig. 2. (a) Forces in a hinge joint generated by two antagonistic muscles plotted over joint angle for maximal activation. The dashed lines indicate force contributions corresponding to three different joint positions. (b) Joint stiffness plotted over joint angle for the setup introduced in (a) for perfect co-activation $\left(a_{1}=a_{2}\right)$. Maximum co-activation is depicted in black.

damping in a musculoskeletal system is investigated and it is stated that the velocity-dependent damping may play an important role in reducing the destabilizing effect due to reflex delays. Muscle viscosity depends on the steepness of the Force-Velocity relation [27] and increases with increasing muscle activation. Therefore, joint viscosity depends on the joint angle, its velocity and the muscle activations. The derivation of joint viscosity for the setup in analysis is given in the Appendix B.

\section{Stability analysis for the antagonistic musculoskeletal setup with constant activations}

In section 5, a bio-inspired control scheme for the concurrent control of position and stiffness will be introduced. This scheme makes use of stiffness regulation through co-contraction. In the present section, the aspect of stability of muscle-driven joints is briefly revisited as preparation for our controller setup. It has been shown that muscles alone mounted in an antagonistic setup provide an intrinsic stability effect at joint level [28, 29]. In fact, spring-like and viscous-like muscle properties produce zero-delay resistive forces against external perturbations [30]. Only for strong perturbations, additional control mechanisms might be required to damp the oscillations and reduce the settling time [9]. In particular, as it has been pointed out in [31], although energetically expensive, high coactivations have a stabilizing effect on the joint. Our stiffness control scheme also integrates this aspect and, with the objective to show its effect on the antagonistic joint described above, the stability properties are analyzed when the system is subject to perturbations. Firstly, the mathematical condition for finding the equilibrium point is discussed. Then, different cases for constant activations supplied to the muscles are studied. Simulation results are shown for the following three situations: low, medium and high muscle activations. Consider the nonlinear system:

$$
\dot{\mathbf{x}}=\mathbf{f}(\mathbf{x}, \mathbf{t}) \quad, \quad \mathbf{x}\left(\mathbf{t}_{\mathbf{0}}\right)=\mathbf{x}_{\mathbf{0}} \in \mathfrak{R}^{n}
$$

where $\mathbf{x}$ is the state of the system, $\mathbf{x}_{\mathbf{0}}$ is its initial condition and $t \geq 0$. $\mathrm{x}^{*}$ is said to be an equilibrium point of (10) if $\mathbf{f}\left(\mathbf{x}^{*}, \mathbf{t}\right) \equiv 0$ for all $t \geq 0$. For the system under analysis the equilibrium points can be obtained by studying the solutions of (7) which correspond to the study of the following system:

$$
\begin{cases}\omega & =0 \\ \tau\left(a_{1}, a_{2}, \alpha^{*}, \omega\right)-\tau_{\mathrm{G}}\left(\alpha^{*}\right)-\tau_{\mathrm{L}} & =0\end{cases}
$$

where $\alpha^{*}$ is the equilibrium position. It is important to notice that, once the activation $a_{1}$ is selected and the load torque $\tau_{\mathrm{L}}$ is defined, the system (11) can be solved with respect to the only unknown $a_{2}$ with the constraint

$$
0 \leq a_{2} \leq 1 .
$$

For the antagonistic joint setup described above, the unperturbed equilibrium position is defined as 

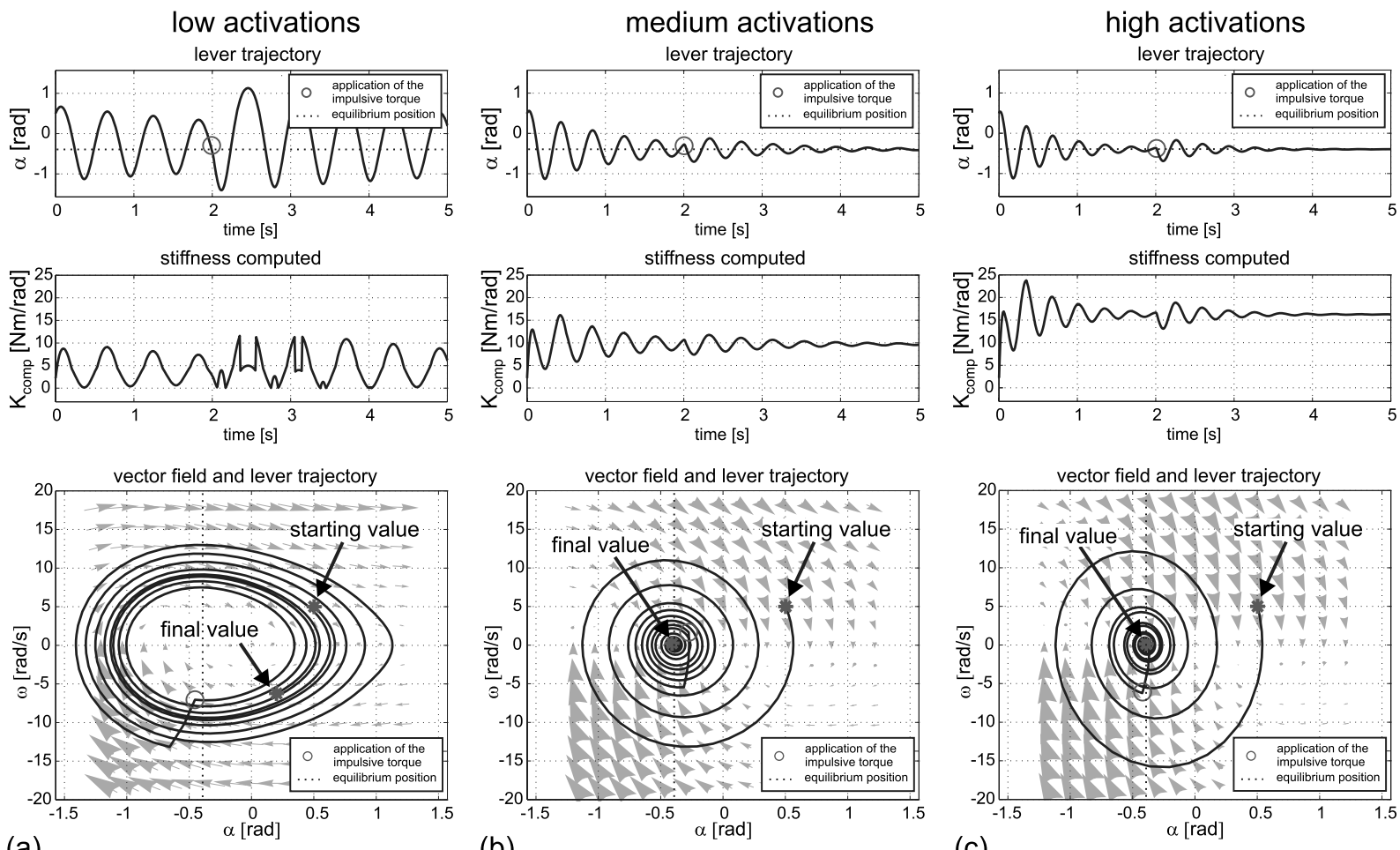

(c)

Fig. 3. Stability analysis of the hinge joint loaded by external forces for three different activation levels. In the top of the figure the lever trajectory and the stiffness computed are plotted over time. In the bottom are depicted the vector field and the lever trajectory. (a) depicts the simulation results in the case of muscles low activated. The equilibrium position is stable but oscillations are high. At $t=2 \mathrm{~s}$ an impulsive torque is applied to the lever which increases limb oscillations. (b) same situation as (a) but for medium activations. Oscillations are more damped and the impulsive torque has a slighter perturbing effect on the limb. (c) same as (b) but for higher activations. High joint stiffness provides a stabilizing effect at joint level.

$\alpha^{*}=-0.4 \mathrm{rad}$. Three simulations with increasing values of $a_{1}\left(a_{1}=0, a_{1}=0.5, a_{1}=1\right)$ are performed while a mass $\left(M_{\mathrm{L}}\right)$ is attached to the lever to increase the inertia and an external load force $\left(F_{\mathrm{L}}\right)$ is applied at its end. It can be shown through linearization around the equilibrium point that $\alpha^{*}$ corresponds to a stable equilibrium in all analyzed cases. Each simulation runs for 5 seconds and starts from the initial condition $\left(\alpha_{0}, \omega_{0}\right) \equiv(0.5,5)$. Furthermore, at $t=2 \mathrm{~s}$ the joint is subjected to an impulsive torque (amplitude $20 \mathrm{Nm}$ and duration $20 \mathrm{~ms}$ ) which simulates a disturbance.

By solving the system (11) for each value $a_{1}$, a corresponding value for $a_{2}$ is obtained such that the equilibrium position is kept at $\alpha^{*}$. Table 3 in the Appendix $\mathrm{C}$ reports the activations and the simulation parameters used in the analysis. Figure 3 depicts the simulation results in the following three cases: low activations, medium activations and high activations. The plots at the top of the figure show the trajectory of the lever over time and the corresponding stiffness computed using Equation (9). The bottom plots in Fig. 3 show the vector field together with the trajectory of the lever from the starting value $\left(\alpha_{0}, \omega_{0}\right)$ to the final value reached after 5 seconds of simulation. For low activations (Fig. 3a) a low stiffness is generated and the lever, although stable, is slightly damped and oscillates around the asymptotic equilibrium position. At $t=2 \mathrm{~s}$ the impulsive torque is applied and the system keeps a stable behavior although oscillations around the equilibrium position become higher. Figure $3 \mathrm{~b}$ depicts the simulation results in the case of medium activations. Stiffness is higher and oscillations are more damped around the equilibrium with respect to the case before. The application of the impulsive torque at $t=2$ has a small effect on the lever as the higher stiffness produces a stabilizing effect on the joint. In the case of high activations (Fig. 3c) the system reaches the equilibrium position 
after 2 seconds with damped oscillations. The impulsive external torque produces a small perturbation of the position of the lever since joint stiffness is high.

\section{Bio-inspired control system design}

In this section, a bio-inspired concurrent control of position and stiffness for the antagonistically actuated hinge joint as described in section 2 is designed. The complete control scheme can be divided in two separate mechanisms. A position controller, which is responsible for generating the necessary torque in order to reach the desired position, and a stiffness controller which has the task to reach the desired stiffness and adequately compensate changes in stiffness due to the position controller action. As it is claimed in [23], the implementation of an intelligent stiffness controller provides the mechanical system with the ability to execute tasks also with unstable loads. The only feedback based position control could in fact not be adequate to cope with such tasks because of the time delay [23, 32]. In this work, the position controller relies on reciprocal activation of antagonistic muscles. The stiffness controller implements a co-activation strategy. Therefore, the control scheme implemented here is based on the following idea: When a perturbing torque is acting on the joint, muscles change their lengths and as consequence a reciprocal activation is sent to counter-act the load together with a co-activation signal in order to adequately stiffen the joint and stabilize the contact.

Figure 4 depicts the complete setup with the Position/Stiffness Controller and the Antagonistic Joint. In the following subsections, the bio-inspired position and stiffness control mechanisms are described.

\subsection{Position controller}

Figure 5 depicts the position controller which integrates the model of physiological properties and motor-neurons (MNs). During voluntary control of muscle length, the $\alpha$-MN and the $\gamma$-MN (not shown) receive a reference value corresponding to the expected shortening of the muscle during the movement $\left(\alpha^{*}\right.$ $\gamma^{*}$ co-activation) $[33,34]$. The command $\alpha^{*}$ is sent to the $\alpha$-MN while $\gamma^{*}$ is responsible for producing a shortening in the muscle spindle which corresponds to the desired trajectory at joint level. In an antagonistic setup, depending on the desired direction of motion, one muscle is activated and contracts while the other relaxes. The command which activates either $M_{1}$ or $M_{2}$ comes from the reciprocal inhibition block through the $I_{a}$-interneuron $\left(I_{a}\right.$-IN). The muscle spindle is modeled in this work as transducer, comparator and controller for the angular position. The $\alpha-M N$ is the command center that activates the muscle. The Renshaw cell is responsible for controlling the

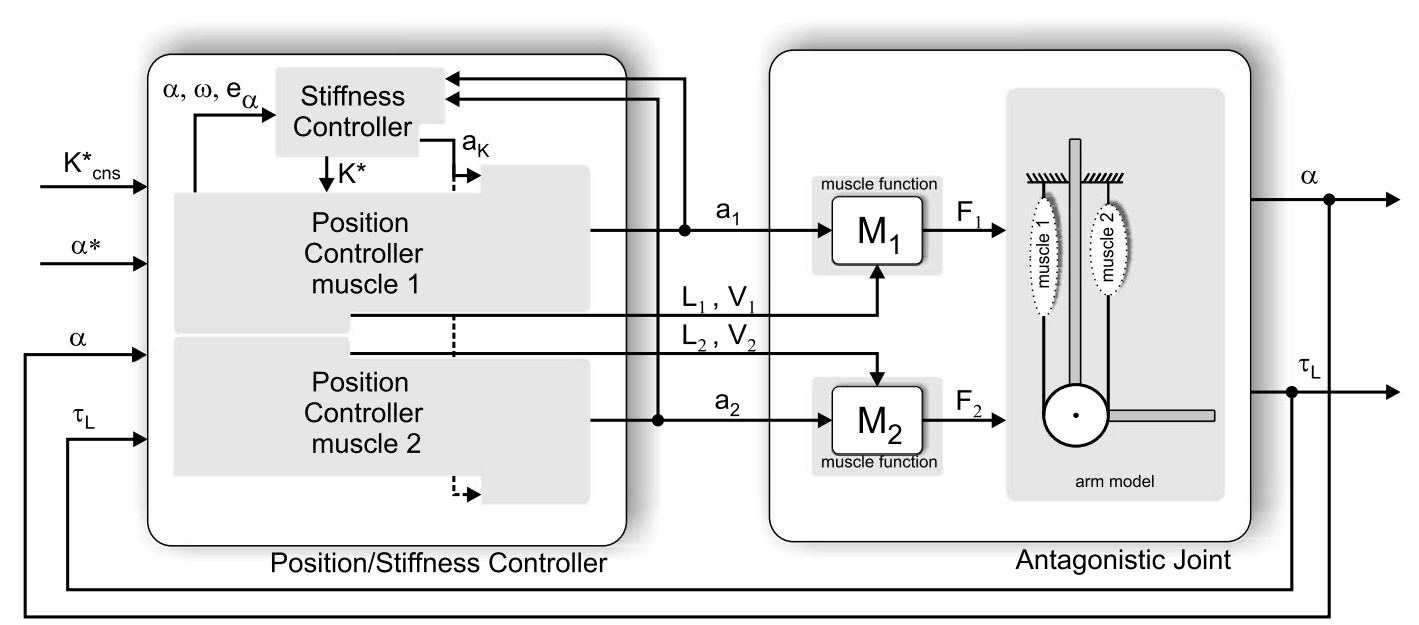

Fig. 4. Schematic view of the simultaneous Position/Stiffness Controller acting on the Antagonistic Joint. On the right side, two muscles ( $M_{1}$ and $\left.M_{2}\right)$ are placed in an antagonistic joint setup (arm model). Forces $F_{1}$ and $F_{2}$ are generated based on the actual activation levels $\left(a_{1}\right.$ and $\left.a_{2}\right)$ and muscle lengths/velocities $\left(L_{1}, V_{1}, L_{2}, V_{2}\right)$. The position controllers on the left side activate either the agonistic or the antagonistic muscle in dependence of the joint position error. The stiffness controller at the top generates the same activation $\left(a_{\mathrm{k}}\right)$ for both muscles (co-activation). 


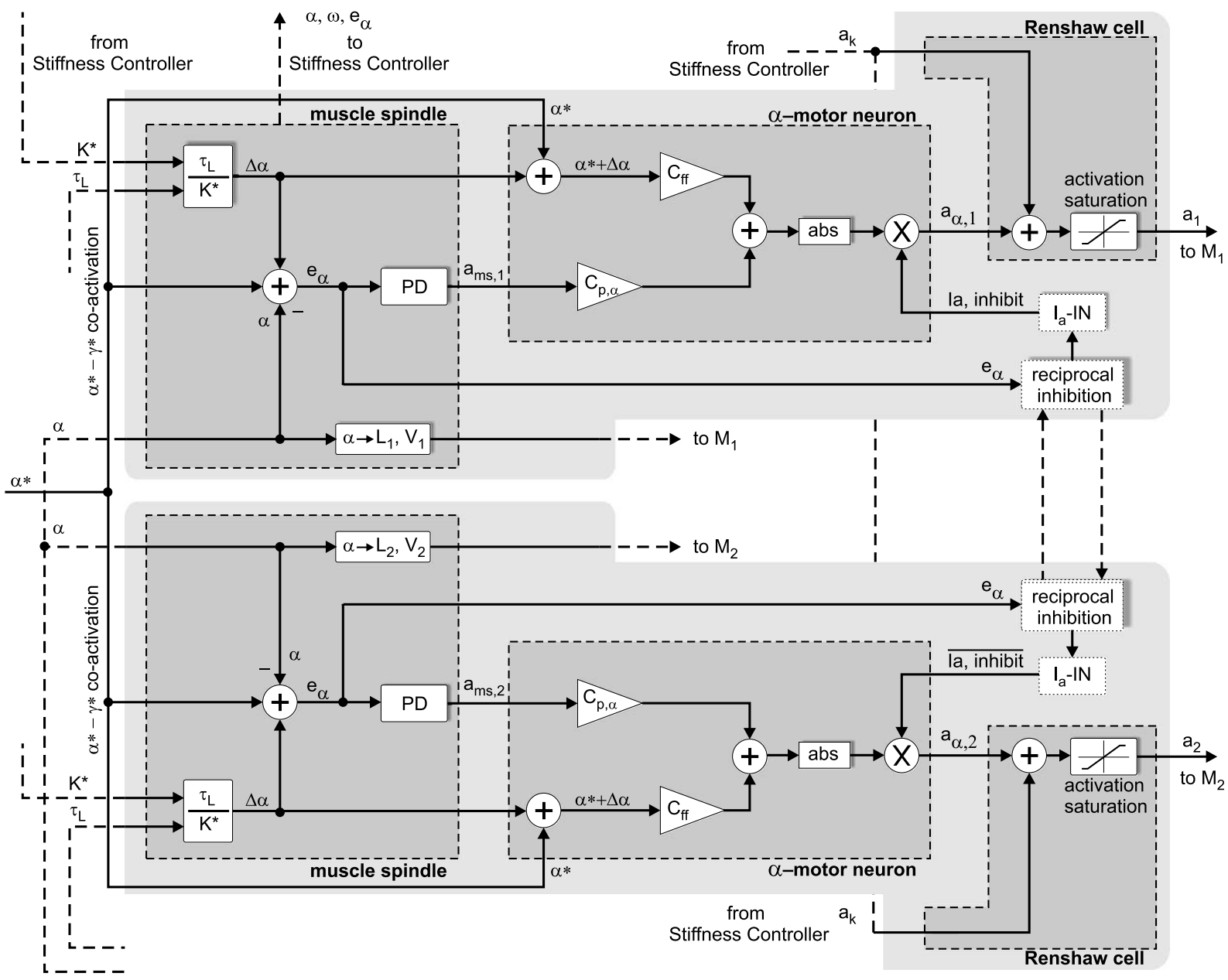

Fig. 5. Schematic of the bio-inspired position controller for the antagonistic joint setup. In dependence of the joint position error $\left(e_{\alpha}\right)$ the reciprocal inhibition block activates, through the $I_{a}$-IN, either the position controller on the top (muscle 1 ) or the one on the bottom (muscle 2 ). A reference value is sent to both muscle spindle and $\alpha$-motor neuron $\left(\alpha^{*}-\gamma^{*}\right.$ co-activation). A position offset $(\Delta \alpha)$ is generated when a load ( $\left.\tau_{L}\right)$ is applied to the output and added to the set points. The muscle spindle works as transducer, comparator and controller for the joint position. The control signal generated in the muscle spindle is amplified by the $\alpha$-motor neuron and added to a feed-forward position signal. The resulting activation is then summed to the activation coming from the stiffness controller $\left(a_{\mathrm{k}}\right)$, saturated to 1 (Renshaw cell) and sent to the muscle.

co-activation (through the stiffness controller which is described in subsection 5.2) and for limiting the total activation to the muscle (activation saturation block). The force feedback is realized with the measure provided by the Golgi tendon organ (GTO). In the following, the description of each subunit pointed out here is given.

\subsubsection{Muscle spindle}

The muscle spindle is a specialized muscle receptor which, modeled as a sensor, measures the actual muscle length $(L)$ and its rate of change $(V)$. It is also a transducer as it transmits those measures to the CNS. Furthermore, it is a controller of the muscle length. In particular, the muscle spindle reacts to any muscle stretch by increasing the firing rate (stretch-reflex loop). The firing rate depends on the muscle-length and also on the change of the muscle-length. When an external load is applied to the joint, the muscle is stretched and, as a consequence, the muscle spindle increases the firing rate to the $\alpha$-MN in order to counteract the load. With the objective to realize a compliant 
behavior when a load is applied to the system, a position offset $(\Delta \alpha)$ defined as

$$
\Delta \alpha=\frac{\tau_{L}}{K^{*}}
$$

is added to the set point (where $\tau_{L}$ is the load torque and $K^{*}$ is the desired stiffness). In this way the desired stiffness modulates how much of the perturbation is visible on the position. Therefore, the muscle spindle and the reflex connections form a feedback system to control joint position (stretch-reflex). Those mechanisms are modeled in Fig. 5 as a feedback control loop with a proportional-derivative (PD) controller. When the joint position error $e_{\alpha}$ is defined as the difference between compliant desired position $\left(\alpha^{*}+\Delta \alpha\right)$ and actual position $(\alpha)$

$$
e_{\alpha}=\alpha^{*}+\Delta \alpha-\alpha
$$

the firing rate of the muscle spindle is

$$
\begin{aligned}
& a_{\mathrm{ms}, 1}=c_{\mathrm{p}, 1} \cdot e_{\alpha}(t)+c_{\mathrm{d}, 1} \cdot \frac{d}{d t} e_{\alpha}(t) \\
& a_{\mathrm{ms}, 2}=c_{\mathrm{p}, 2} \cdot e_{\alpha}(t)+c_{\mathrm{d}, 2} \cdot \frac{d}{d t} e_{\alpha}(t)
\end{aligned}
$$

with $c_{\mathrm{p}}$ and $c_{\mathrm{d}}$ being the proportional and derivative gains of the position controller (PD-controller in the muscle spindle box, the index refers to the muscle).

\subsection{2. $\alpha$-motor neuron and reciprocal inhibition}

The muscle spindle does not directly cause the muscle to contract (its firing rate $a_{\mathrm{ms}}$ is too small to produce a muscle contraction [11]). Instead, it causes activation of the $\alpha-\mathrm{MN}$ which is responsible for direct muscle activation. Before the activation signal reaches the muscle, $a_{\mathrm{ms}}$ is amplified in the block $c_{\mathrm{p}, \alpha}$. Additional inputs of the $\alpha$-MN are the feed-forward signal $\alpha^{*}$ and the position offset $\Delta \alpha$ which is added to $\alpha^{*}$. While $\alpha^{*}$ is a pure feed-forward signal for the position, the additional position offset $\Delta \alpha$ implements the zero feedback delay due to spring-like muscle behavior when a load is applied [35]. The sum of the two signals is then multiplied by a scaling factor ( $C_{\mathrm{ff}}$ block) and added to the activation due to the stretch-reflex.

In order to reach the desired joint position the CNS activates either the agonistic or the antagonistic muscle (reciprocal activation). The activated muscle shortens while the other relaxes. This mechanism is controlled by the reciprocal inhibition block in which an inhibitory signal is transmitted to the $\alpha$-MN of the muscle that has to relax. The $I_{a}$ inhibitory signal is generated by a disynaptic pathway involving an $I_{a}$ inhibitory interneuron ( $I_{a}$-IN block). The variable $I_{\mathrm{a}, \text { inhib }}$ indicates the $I_{a}$ inhibitory signal and is defined as

$$
I_{\mathrm{a}, \text { inhib }}= \begin{cases}1 & e_{\alpha}<0 \\ 0 & e_{\alpha} \geq 0 .\end{cases}
$$

Based on the mechanisms described above, the firing rate of each $\alpha-\mathrm{MN}$ is

$a_{\alpha, 1}=\left|c_{\mathrm{ff}, \alpha} \cdot\left(\alpha^{*}+\Delta \alpha\right)+c_{\mathrm{p}, \alpha} \cdot a_{\mathrm{ms}, 1}\right| \cdot I_{\mathrm{a}, \text { inhib }}$
$a_{\alpha, 2}=\left|c_{\mathrm{ff}, \alpha} \cdot\left(\alpha^{*}+\Delta \alpha\right)+c_{\mathrm{p}, \alpha} \cdot a_{\mathrm{ms}, 2}\right| \cdot \overline{I_{\mathrm{a}, \text { inhib }}}$

for which either $M_{1}$ or $M_{2}$ is activated to generate a torque in the desired angular direction and with $c_{\mathrm{ff}, \alpha}$ being the feed-forward scaling gain and $c_{\mathrm{p}, \alpha}$ being the proportional (amplification) gain of the position controller.

\subsubsection{Golgi tendon organ and Renshaw cell}

The Golgi tendon organ is a mechanoreceptor located between muscle and tendon and measures the tension. In our model, all GTOs are assumed to provide the total force at joint level as a feedback signal $\tau_{L}$ to the CNS [36].

The Renshaw cell plays two important roles. Firstly, it receives information from the $\alpha$-MN on its firing rate $\left(a_{\alpha}\right.$ signal) and has the objective to reduce it thus acting as limiter [37]. Secondly, it produces an inhibitory signal in order to regulate the strength of reciprocal inhibition (recurrent inhibition). In this case the Renshaw cell inhibits the $I_{a}$-IN, which decreases its activity (disinhibition) and as a consequence the coactivation of antagonistic muscles is obtained. The first part is modeled with an activation saturation block in Fig. 5. The second part is modeled as additional activation of the muscles provided by a stiffness controller. The contribution of the stiffness controller is depicted as a sum block before the saturation in the Renshaw cell box. According to [38], despite its energetic cost, the CNS uses co-contraction as a strategy to increase accuracy during movements and minimize the perturbing effects of external loads. For that reason the same signal $a_{\mathrm{K}}$ is sent to both muscles. The description of the stiffness controller is given in the next subsection.

\subsection{Stiffness controller}

In [39] it is claimed that Purkinje cells activity could use reciprocal inhibition to control the tone of antagonistic muscles providing the control of switching 


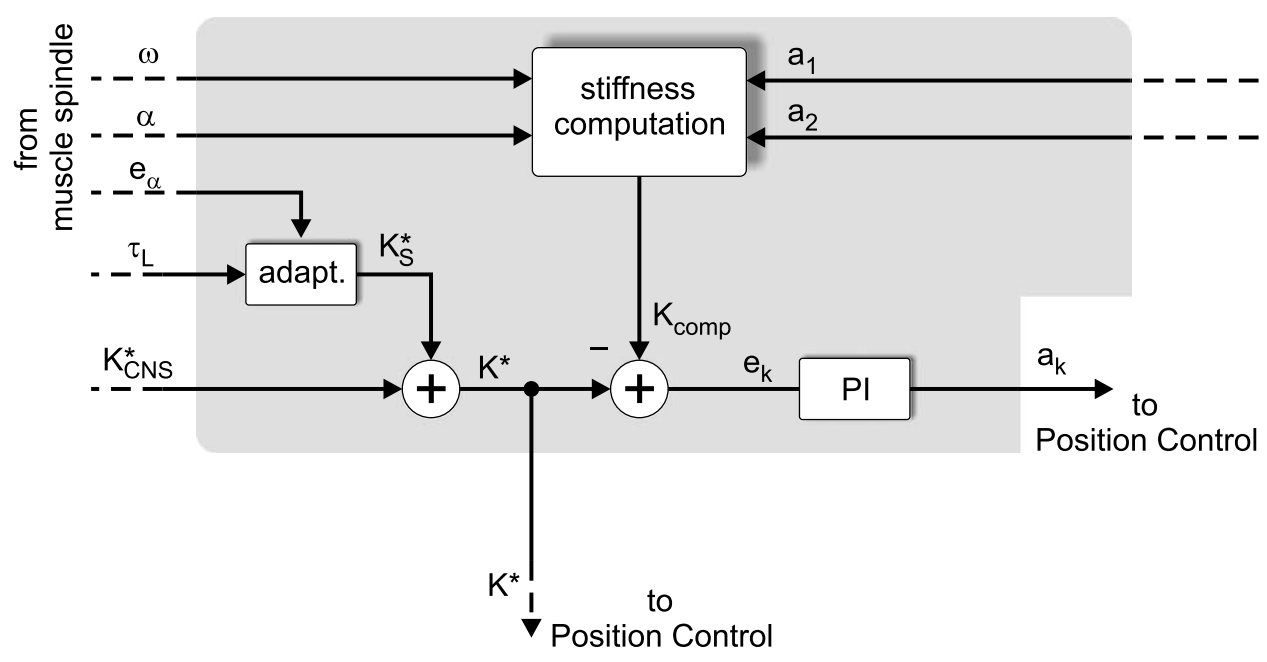

Fig. 6. Schematic of the stiffness controller. The block stiffness computation computes the actual stiffness based on the joint angle ( $\alpha$ ), velocity $(\omega)$ and activations $\left(a_{1}\right.$ and $a_{2}$ ). The desired stiffness is the sum of two parts: $K_{\mathrm{CNS}}^{*}$ (stiffness reference "from the brain") and $K_{\mathrm{S}}^{*}$ (additional stiffness reference generated by an adaptive block, adapt.). The stiffness controller is a PI and its output ( $\left.a_{k}\right)$ acts on both muscles (co-activation).

between reciprocal activation and co-activation. The stiffness controller described in this work implements the decrease of reciprocal inhibition in the antagonistic muscles [40] and thus allows co-contraction. As it was observed in [23], so far, no real stiffness controller can be found in biological systems since experiments are designed to distinguish between force control and position control mechanisms. In this work, an explicit stiffness controller is proposed and its possible connection to the bio-inspired position control mechanism (as introduced before) is explained. The use of an explicit stiffness controller is also important for the discussion of stability issues (section 4).

Figure 6 depicts this stiffness controller. The computation of the actual joint stiffness $K_{\text {comp }}$ is realized in the block stiffness computation which implements expression (9) and makes use of the actual activation values $a_{1}$ and $a_{2}$, joint position $\alpha$ and velocity $\omega$. As shown in section 4 , when controlling the position, the stiffness is the key to concurrently control stability [10]. For this purpose, together with actual joint position and velocity, the muscle spindle provides also the position error $e_{\alpha}$ which is used as a stability measure [10] in an adaptive scheme. The adaptive control block (adapt.) receives $e_{\alpha}$ and $\tau_{L}$ and has the goal to increase the desired stiffness coming from the CNS when an external load is applied. Therefore, the desired stiffness is composed of two parts:

$$
K^{*}=K_{\mathrm{CNS}}^{*}+K_{\mathrm{S}}^{*}
$$

where $K_{\mathrm{CNS}}^{*}$ is the reference of stiffness "coming from the brain" while $K_{\mathrm{S}}^{*}$ is the additional stiffness coming from the adaptive block and is defined as

$$
K_{\mathrm{S}}^{*}= \begin{cases}c_{\mathrm{S}} \cdot \int_{t-T}^{t}\left|e_{\alpha}(t)\right| d t & \text { if } \tau_{L} \neq 0 \\ 0 & \text { otherwise }\end{cases}
$$

with $c_{\mathrm{S}}$ defining the speed of adaptation and $\mathrm{T}$ the integration time. In this way when the position error becomes high, the desired stiffness increases and as a consequence muscles are more co-activated ensuring a higher stability and accuracy with respect to external perturbations for the joint [9]. The stiffness controller is of the proportional-integral type (PI). Therefore, starting from the stiffness error

$$
e_{\mathrm{k}}=K^{*}-K_{\mathrm{comp}}
$$

the controller generates the stiffness control value

$$
a_{\mathrm{k}}=c_{\mathrm{p}, \mathrm{k}} \cdot e_{\mathrm{k}}+c_{\mathrm{i}, \mathrm{k}} \cdot \int e_{\mathrm{k}} d t
$$

with the proportional and integral controller gains $c_{\mathrm{p}, \mathrm{k}}$ and $c_{\mathrm{i}, \mathrm{k}}$. Both muscles receive the same activation value $a_{\mathrm{k}}$ (co-activation). 


\section{Simulation results for the bio-inspired controller}

For the hinge joint described in section 2, two different tasks are simulated in order to verify the performances of the bio-inspired position/stiffness control scheme introduced in the previous section to show that the results comply with those obtained in biological experiments. In the first simulation, a simultaneous control of position and stiffness is tested when a sinusoidal load is applied at the lever. Both the activations that control position and stiffness are separately shown and discussed. In the second simulation, the controller is tested in the case of contact with an obstacle and in the presence of different perturbing forces. Depending on the frequency of the perturbation the stiffness is adjusted by the adaptive control mechanism. For low frequency perturbations the joint is compliant, while for rapid perturbations joint stiffness increases and the effect of the external force is reduced [25]. A medium level of stiffness is desired in both tests as it is considered an optimal value during joint movement [24]. The control scheme and the musculoskeletal joint setup were modeled in Matlab/Simulink ${ }^{\circledR} 7.11$ (The MathWorks Inc. Natick, MA, USA). Simulation parameters for the controllers are given in Table 4 in the Appendix C.

\subsection{Compliant concurrent position/stiffness control for an external sinusoidal load}

Figure $7 \mathrm{a}$ shows the simulation results of the concurrent control of position and stiffness for a compliant behavior when an external load is applied. The desired position is constant ( $\left.\alpha^{*}=-0.3 \mathrm{rad}\right)$. First, the desired stiffness is constant $\left(K^{*}=5 \mathrm{Nm} / \mathrm{rad}\right)$ but at $t=3 \mathrm{~s}$ it doubles its value $\left(K^{*}=10 \mathrm{Nm} / \mathrm{rad}\right)$. At $t=1 \mathrm{~s}$, a sinusoidal load $\tau_{L}$ of amplitude $2 \mathrm{Nm}$ and frequency $4 \mathrm{rad} / \mathrm{sec}$ is applied to the extremity of the lever arm and the compliant desired position $\left(\alpha^{*}+\Delta \alpha\right)$ is modified. As the position error increases due to the load applied, the adaptive block increases the desired stiffness as long as the error remains large. When the error reduces, the stiffness decreases back to the desired value. At $t=3 \mathrm{~s}$ the desired stiffness doubles which results in an increase of co-activation. As a consequence, the joint compliance is modified and the effect due to $\tau_{L}$ is reduced $[8,13]$. The activations are shown in Fig. 7b. The position controller reduces the error on the position by activating either muscle $1\left(a_{\alpha, 1}\right)$ or muscle $2\left(a_{\alpha, 2}\right)$ implementing a reciprocal activation strategy, while the stiffness controller sends the same activation value $a_{\mathrm{k}}$ to both muscles, thus implementing a co-activation strategy. Muscles receive the sum of the two activations.

\subsection{Position control and compliance adaptation with respect to different external perturbations}

In the previous example, the control scheme was tested for a sinusoidal load perturbation applied to the lever for two different values of stiffness to investigate the interaction of the separated position and stiffness controllers. In the second test depicted in Fig. 8, the lever arm was commanded to follow a sinusoidal trajectory till $t=6 \mathrm{~s}$ and then to keep a constant position $\left(\alpha^{*}=-0.3 \mathrm{rad}\right)$. The desired stiffness is constant $\left(K^{*}=3 \mathrm{Nm} / \mathrm{rad}\right)$. From $t=2 \mathrm{~s}$ to $t=4 \mathrm{~s}$ an obstacle ("collision") in the form of a stiff spring $\left(K_{x}=10 \cdot 10^{3} \mathrm{~N} / \mathrm{m}\right)$, which is mechanically coupled with the tip of the lever, forces the arm to remain at $\alpha=-0.1 \mathrm{rad}$. Stiffness increases as the "collision" takes place and grows until the disturbing effect is overcome, then it goes back to the desired value. At $t=4 \mathrm{~s}$ the obstacle is removed and the lever returns to the desired trajectory. From $t=8 \mathrm{~s}$ to $t=12 \mathrm{~s}$ a low frequency sinusoidal load $\tau_{L}$ (amplitude $2 \mathrm{Nm}$ and frequency $4 \mathrm{rad} / \mathrm{s}$ ) is applied to the extremity of the lever arm. The generated compliant behavior (stiffness remains low) makes sure that the link follows the external load. From $t=12 \mathrm{~s}$ to $t=16 \mathrm{~s}$ a high frequency sinusoidal perturbation $\tau_{L}$ (amplitude $2 \mathrm{Nm}$ and frequency $40 \mathrm{rad} / \mathrm{s}$ ) is applied to the extremity of the lever arm. In this case the adaptive controller increases the desired stiffness in order to stabilize the system and to reduce the disturbance on the position due to $\tau_{L}$. From $t=16 \mathrm{~s}$ to $t=20 \mathrm{~s}$ two torque pulses load $\tau_{L}$ (amplitude $-5 \mathrm{Nm}$ and duration $20 \mathrm{~ms}$ ) are applied to the extremity of the lever arm. Also in this case, desired joint stiffness increases which produces a stabilizing effect for the limb.

\section{Conclusion}

Biological systems are antagonistically actuated and are able to adapt the joint behavior to the requirements of different contexts by means of controllable 


\section{Compliant Position/Stiffness Control}
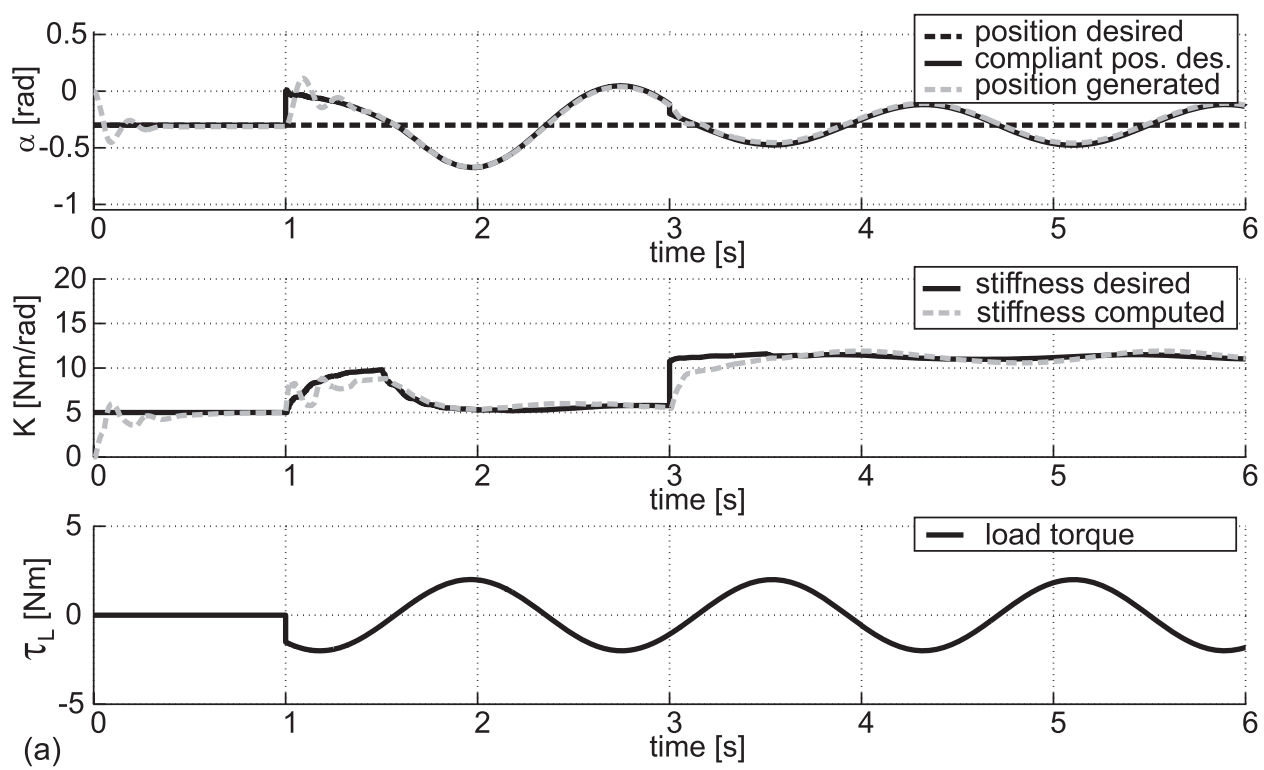

(a)
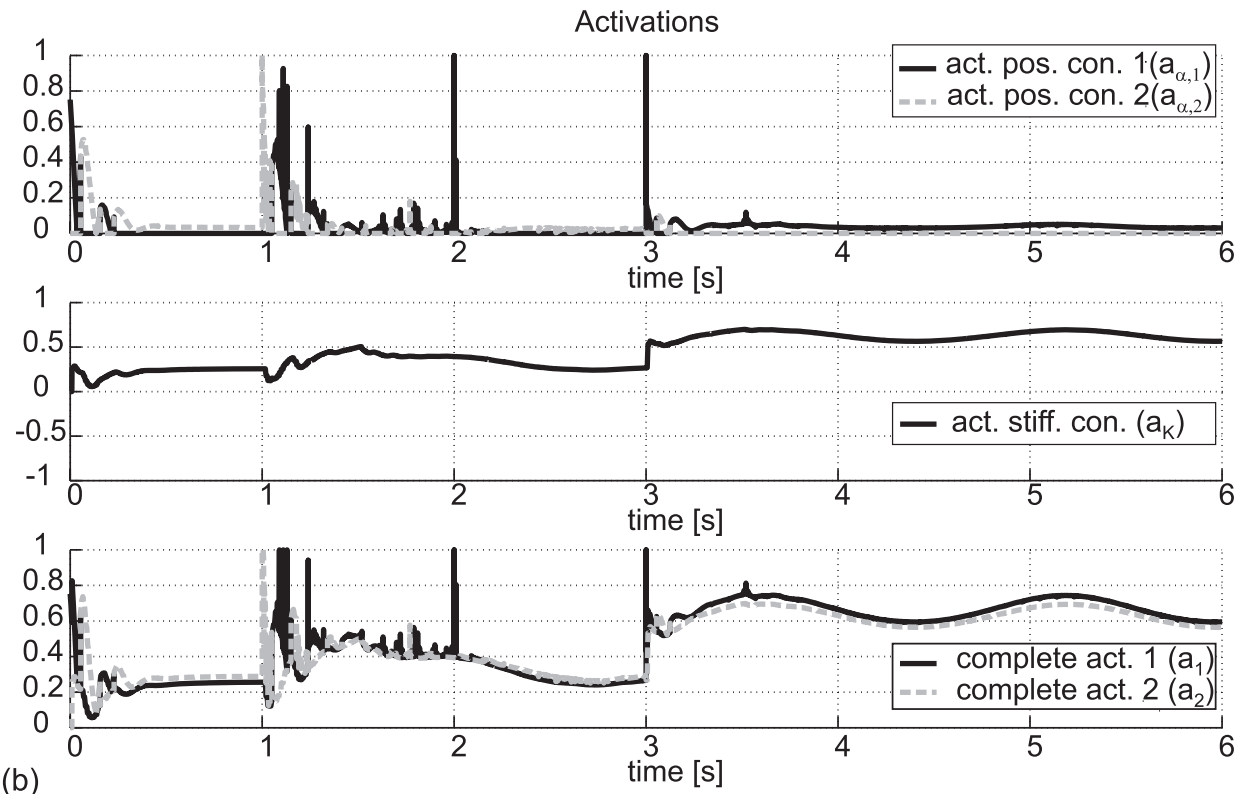

Fig. 7. Simulation results of the concurrent Position/Stiffness Controller with a compliant behavior for an antagonistic muscle pair in a hinge joint setup. (a) A sinusoidal torque is applied to the extremity of the lever at $t=1 \mathrm{~s}$. The desired compliant position is modified based on the actual stiffness. At $t=3$ the desired stiffness doubles its value and the desired compliant position is modified accordingly. (b) Activations for the results in (a) are depicted. The position controller activates only one muscle at a time (either $a_{\alpha, 1}$ or $a_{\alpha, 2}$ is different than zero). The stiffness controller acts on both muscles with the same activation value $a_{\mathrm{K}}$. Muscles receive the complete activation as the sum of the two $\left(a_{1}=a_{\alpha, 1}+a_{\mathrm{K}}\right.$ and $a_{2}=a_{\alpha, 2}+a_{\mathrm{K}}$.

compliance. The results of this work suggest that the proposed bio-inspired control scheme is able to successfully regulate joint position and compliance simultaneously in an antagonistically actuated joint setup. Additionally, the control of compliance is achieved through stiffness adaptation for different 
Compliant Position Control subject to different load conditions
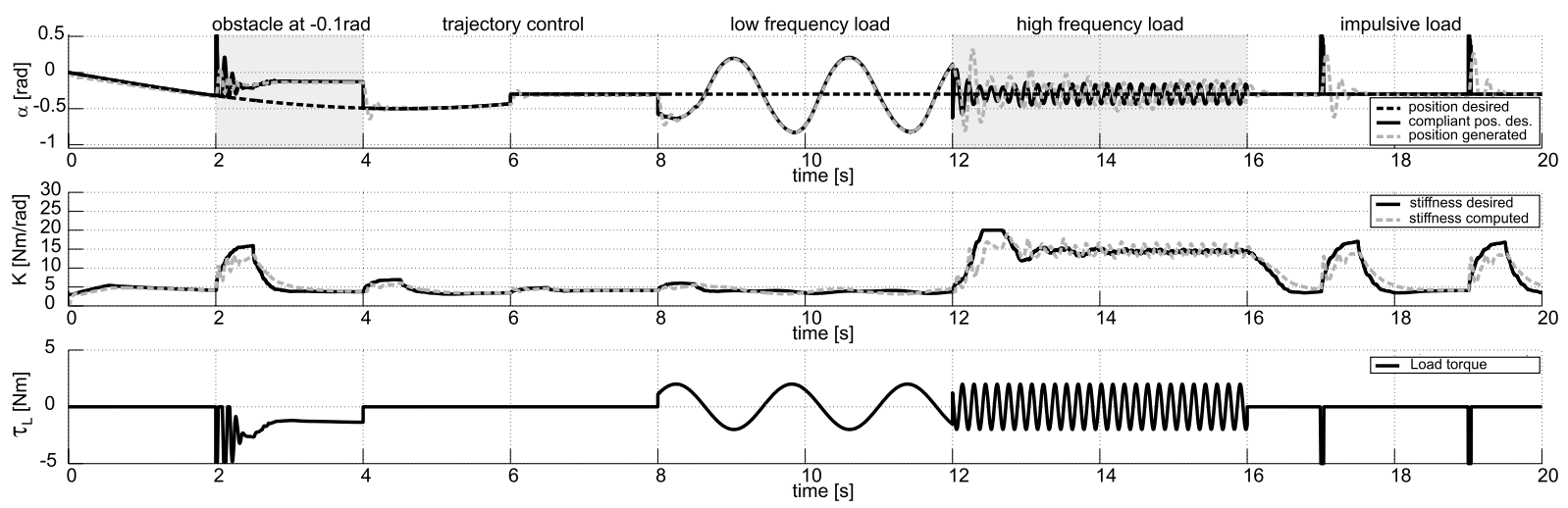

Fig. 8. Simulation results of the position control scheme and compliance adaptation in presence of different perturbing torques. Depending on the perturbation, joint stiffness adapts producing a stabilizing effect at joint level.

external perturbations and ensures stability. Two muscles were arranged in an antagonistic way and attached to a pulley wheel with a lever arm. Muscle and joint parameters were selected in order to mimic human elbow joint characteristics. Each muscle, seen as a force generator and implemented using the Hill-type model $[14,15]$, was controlled separately. The control schemes were designed based on physiological models and properties of biological systems. In particular, the position controller worked on one muscle at a time activating either the agonistic or the antagonistic muscle (reciprocal activation) according to the desired position. In living systems the intrinsic nonlinear muscle elasticity provides the antagonistic system with a way to regulate stiffness through co-activation. Therefore, the stiffness controller worked on both muscles with the same activation (co-activation of antagonistic muscles). In order to justify the model of the stiffness control scheme, the role of the reciprocal inhibition block which governs the reciprocal activation and acts together with the Renshaw cell for the control of co-activation was discussed. An expression for the joint stiffness was derived in a closed form and used in the stiffness control loop as a measure of the actual stiffness in order to regulate the system compliance. The stability analysis performed through simulations showed that, even for a stable equilibrium, the co-activation level affects the reaction of the joint to external perturbations in terms of oscillations and settling time. This concept leads to the more general question what an optimal joint stiffness should be with respect to different external task conditions.
With the objective to optimize joint compliance with respect to external perturbations, a stability measure based on the position error was used in the implementation of an adaptive block in the stiffness scheme. Adaptation of joint stiffness during perturbations made sure that the system was able to counter-act external forces, keep its stability and reduce the perturbing effects on the joint. The simulation results of the position control and compliance adaptation with respect to different external perturbations demonstrated the ability of the control scheme to simultaneously regulate the position and, in dependence of the perturbation frequency, adapt the joint compliance. For low frequency perturbations the joint is compliant, while for rapid perturbations stiffness increases so as reducing the effect of the external torque on the lever. This result is in line with the human behavior as greater voluntary muscle activation is necessary to move faster or to move against a load [7, 17]. Stiffness also increases when a collision with an obstacle takes place and grows until the disturbing effect due to the obstacle is overcome. Then it reduces back to the desired value.

The results of this work can be compared to [41]. Important differences lie in the use of muscle models, the computation of joint stiffness and the resulting control scheme. Furthermore, in our work, the stiffness of the muscle driven hinge joint based on the intrinsic properties determined by the nonlinear spring-like behavior of muscles was analyzed.

For the model of the biological systems presented, some assumptions have been made which are addressed in the following. The force-length relation of 
the muscle is a quadratic approximation which allows a closed mathematical description of the stiffness.

Fitting of force-length data using e.g. higher orders functions or piecewise linear functions would allow closer numerical approximation. Nevertheless, the simplified expressions as used for the stiffness computation in this work can be easily replaced by Gaussian relations without loss of the stiffness characteristics and controllers abilities discussed here [42, 43].

The muscle model used in this work does not include the contribution of tendon compliance. In fact, it is assumed that the tendon is a serial elastic element of high stiffness (the effect of the tendon on the force-length muscle curve can be neglected in many cases $[6,15])$. The level of abstraction adopted in the present work corresponds to a reasonable compromise between biological details and simplified mechanism and allowed us to examine physiologically based control strategies in simulation for posture control and compliance regulation.

In the future, applications which combine technical and biological systems in fields like prosthetics and orthotics may take advantage of the control schemes modeled in this work thanks to the mutual embodiment of drive oriented control signals and user oriented biological commands.

The present study was conducted based on computer simulations. Future works will focus on the implementation of the bio-inspired control laws designed and tested in this work in a technical joint drive and in a multi-joint robot limb. With the objective of realizing a bio-equivalent technical actuation system, a transfer of biological properties to technical systems would be necessary. The sum of all muscle torques pulling on the joint could be replaced by a single net torque which should be then generated either by one actuator or by a set of actuators. In either case, if a bio-equivalent behavior is wanted to be obtained, while controlling the actually used actuator, its specific dynamic effects should be completely compensate, e.g. through feedback linearization, thus realizing the desired muscle dynamics.

\section{Appendix}

\section{Appendix A. Moment of inertia of the forearm about the axis of rotation}

The setup of the biomechanical system is given in Fig. 1b. The forearm has a length of $l=0.35 \mathrm{~m}$ and is considered to be a uniform stick so that its center of mass is at the geometrical center. The mass is $m=1.5 \mathrm{~kg}$. The axis of rotation for the forearm passes through the center of the pulley which corresponds to one end of the stick. From the Parallel Axis Theorem the moment of inertia of the forearm about the axis is given by

$$
J=J_{\mathrm{CM}}+m \cdot d^{2}
$$

where $J_{\mathrm{CM}}$ is the moment of inertia of the forearm if the rotation axis was passing through its center of mass, and $d$ is the displacement of the axis from the center of mass, which is $\frac{l}{2}$. Therefore, we get:

$$
\begin{aligned}
J= & \frac{1}{12} \cdot m \cdot L^{2}+m \cdot d^{2} \\
= & \frac{1}{12} \cdot(1.5 \mathrm{~kg})(0.35 \mathrm{~m})^{2} \\
& +(1.5 \mathrm{~kg})\left(\frac{0.35}{2} \mathrm{~m}\right)^{2}=0.06125 \mathrm{~kg} \mathrm{~m}^{2}
\end{aligned}
$$

So the rotational inertia of the forearm about the axis is $0.06125 \mathrm{~kg} \mathrm{~m}^{2}$.

\section{Appendix B. Derivation of the expression for the joint viscosity}

The expression of the viscosity for the antagonistic joint in this work can be obtained by calculating the first derivative of the net joint torque $\tau$ with respect to the joint velocity $\omega$

$$
\begin{aligned}
& B=\left.-\frac{\partial \tau}{\partial \omega}=-\frac{\partial\left(F_{M, 2} \cdot r\right.}{\partial \omega}-F_{M, 1} \cdot r\right) \\
&=-\frac{\partial\left[\left(F_{L a}\left(\Delta L_{\mathrm{eff}, 2}\right) \cdot F_{V}\left(V_{2}\right) \cdot a_{2}+F_{L p}\left(\Delta L_{\mathrm{eff}, 2}\right)\right) \cdot F_{\max , 2} \cdot r\right]}{\partial \omega} \\
&+\frac{\partial\left[\left(F_{L a}\left(\Delta L_{\mathrm{eff}, 1}\right) \cdot F_{V}\left(V_{1}\right) \cdot a_{1}+F_{L p}\left(\Delta L_{\mathrm{eff}, 1}\right)\right) \cdot F_{\max , 1} \cdot r\right]}{\partial \omega} \\
& \text { and by inserting (1), (3), (4), (5) and (6) this yields }
\end{aligned}
$$




$$
\begin{aligned}
& B\left(\alpha, \omega, a_{1}, a_{2}\right) \\
& =a_{1} \cdot F_{\max , 1} \cdot r . \\
& \left(\left\{\begin{array}{ll}
1-\frac{\left(L_{\mathrm{off}, 1}+r \cdot \alpha\right)^{2}}{w_{L 01}^{2}} & \text { if } w_{L 01}>\left|L_{\mathrm{off}, 1}+r \cdot \alpha\right| \\
0 & \text { otherwise }
\end{array}\right.\right. \\
& \cdot\left\{\begin{array}{ll}
\frac{K_{v} \cdot\left(r \cdot \omega+V_{\max }\right)}{\left(-K_{v} \cdot r \cdot \omega+V_{\max }\right)^{2}}+\frac{1}{-K_{v} \cdot r \cdot \omega+V_{\max }} & \text { if } \omega<0 \\
\frac{1-N_{n}-7.56 \cdot K_{v} \cdot N_{n}}{-7.56 \cdot K_{v} \cdot r \cdot \omega-V_{\max }}+\frac{7.56 \cdot K_{v} \cdot\left(r \cdot \omega-N_{n} \cdot r \cdot \omega-7.56 \cdot K_{v} \cdot N_{n} \cdot r \cdot \omega-V_{\max }\right)}{\left(-7.56 \cdot K_{v} \cdot r \cdot \omega-V_{\max }\right)^{2}} & \text { otherwise }
\end{array}\right) \\
& -a_{2} \cdot F_{\max , 2} \cdot r \text {. } \\
& \left(\begin{array}{ll}
1-\frac{\left(L_{\mathrm{off}, 2}-r \cdot \alpha\right)^{2}}{w_{L 02}^{2}} & \text { if } w_{L 02}>\left|L_{\mathrm{off}, 2}-r \cdot \alpha\right| \\
0 & \text { otherwise }
\end{array}\right. \\
& \cdot\left\{\begin{array}{ll}
\frac{-1+N_{n}+7.56 \cdot K_{v} \cdot N_{n}}{7.56 \cdot K_{v} \cdot r \cdot \omega-V_{\max }}-\frac{7.56 \cdot K_{v} \cdot\left(-r \cdot \omega+N_{n} \cdot r \cdot \omega+7.56 \cdot K_{v} \cdot N_{n} \cdot r \cdot \omega-V_{\max }\right)}{\left(7.56 \cdot K_{v} \cdot r \cdot \omega-V_{\max }\right)^{2}} & \text { if } \omega<0 \\
-\frac{K_{v} \cdot\left(-r \cdot \omega+V_{\max }\right)}{\left(K_{v} \cdot r \cdot \omega+V_{\max }\right)^{2}}-\frac{1}{K_{v} \cdot r \cdot \omega+V_{\max }} & \text { otherwise }
\end{array}\right) .
\end{aligned}
$$

\section{Appendix C. Tables with the parameters used during the simulations}

Table 3

Simulation parameters for the stability analysis of the antagonistic pulley joint setup

\begin{tabular}{llcc}
\hline Variable & Description & Value & Unit \\
\hline$\alpha_{0}$ & Initial position & 0.5 & {$[\mathrm{rad}]$} \\
$\omega_{0}$ & Initial velocity & 5 & {$[\mathrm{rad} / \mathrm{s}]$} \\
$\alpha^{*}$ & Equilibrium position & -0.4 & {$[\mathrm{rad}]$} \\
$M_{\mathrm{L}}$ & Load mass & 3 & {$[\mathrm{~kg}]$} \\
$F_{\mathrm{L}}$ & Load force & -15 & {$[\mathrm{~N}]$} \\
$T_{\mathrm{L}}$ & Impulsive load torque & 20 & {$[\mathrm{Nm}]$} \\
\hline Activation & Low act. & Medium act. & High act. \\
\hline$a_{1}$ & 0 & 0.5 & 1 \\
$a_{2}$ & 0.21 & 0.6 & 0.98 \\
\hline
\end{tabular}

Table 4

Simulation parameters for the bio-inspired control strategy of the antagonistic pulley joint setup

\begin{tabular}{llcl}
\hline Variable & Description & Value & Unit \\
\hline$c_{\mathrm{p}, 1}=c_{\mathrm{p}, 2}$ & Proportional gain position contr. & 0.05 & \\
$c_{\mathrm{d}, 1}=c_{\mathrm{d}, 2}$ & Derivative gain position contr. & 0.001 & \\
$c_{\mathrm{p}, \alpha}$ & Proportional gain pos. contr. $(\alpha-\mathrm{MN})$ & 50 & \\
$c_{\mathrm{ff}, \alpha}$ & Scaling gain feed-forward $(\alpha-\mathrm{MN})$ & 0.005 & \\
$c_{\mathrm{p}, \mathrm{K}}$ & Proportional gain stiffness contr. & 0.05 & \\
$c_{\mathrm{i}, \mathrm{K}}$ & Integral gain stiffness contr. & 6 & \\
$c_{\mathrm{S}}$ & Proportional gain adaptive contr. & 0.25 & \\
$T$ & Integration time & 500 & {$[\mathrm{~ms}]$} \\
\hline
\end{tabular}

\section{Acknowledgement}

This work has been supported by the Federal Ministry of Education and Research (BMBF) within the BIONA program (ELAN-project to A.S.), within the Excellence Initiative of the German Research Foundation (DFG Center of Excellence "Cognitive Interaction Technology", EXC277) and by an EU-FP7 grant (ICT2009.2.1, No. 270182 to A.S.).

\section{References}

[1] A. Bicchi and G. Tonietti, Fast and soft arm tactics: Dealing with the safety-performance trade-off in robot arms design and control, IEEE Robotics and Automation Magazine 11(2) (2004), 22-33.

[2] A. Albu-Schäffer, C. Ott and G. Hirzinger, A unified passivitybased control framework for position, torque and impedance control of flexible joint robots, The International Journal of Robotics Research 26(1) (2007), 23-39.

[3] N. Hogan, Adaptive control of mechanical impedance by coactivation of antagonist muscles, Transactions on Automatic Control 29(8) (1984), 681-690.

[4] Y. Yamazaki, T. Ohkuwa, H. Itoh and M. Suzuki, Reciprocal activation and coactivation in antagonistic muscles during rapid goal-directed movements, Brain Research Bulletin 34(6) (1994), 587-593.

[5] Y. Yamazaki, H. Itoh and T. Ohkuwa, Muscle activation in the elbow-forearm complex during rapid elbow extension, Brain Research Bulletin 38(3) (1995), 285-295. 
[6] P.L. Gribble, D.J. Ostry, V. Sanguineti and R. Laboissière, Are complex control signals required for human arm movement? Journal of Neurophysiology 79(3) (1998), 14091424.

[7] M. Suzuki, D.M. Shiller, P.L. Gribble and D.J. Ostry, Relationship between cocontraction, movement kinematics and phasic muscle activity in single-joint arm movement, Experimental Brain Research 140(2) (2001), 171-181.

[8] P. Gribble, L. Mullin, N. Cothros and A. Mattar, Role of cocontraction in arm movement accuracy, $J$ Neurophysiol 89(5) (2003), 2396-2405.

[9] P.C. Chou and B. Hannaford, Study of human forearm posture maintenance with a physiologically based robotic arm and spinal level neural controller, Biological Cybernetics 76(4) (1997), 285-298.

[10] E. Burdet, K.P. Tee, I. Mareels, T.E. Milner, C.M. Chew, D.W. Franklin, R. Osu and M. Kawato, Stability and motor adaptation in human arm movements, Biological Cybernetics 94(1) (2006), 20-32.

[11] W. Hoult and D.J. Cole, A neuromuscular model featuring co-activation for use in driver simulation, Vehicle System Dynamics 46 (2008), 175-189.

[12] D.W. Franklin, E. Burdet, K.P. Tee, R. Osu, C.M. Chew, T.E. Milner and M. Kawato, Cns learns stable, accurate, and efficient movements using a simple algorithm, Journal of Neuroscience 28(44) (2008), 11165-11173.

[13] D. Mitrovic, S. Klanke and S. Vijayakumar, Learning impedance control of antagonistic systems based on stochastic optimization principles, International Journal of Robotics Research 30(5) (2011), 556-573.

[14] A.V. Hill, The heat of shortening and the dynamic constants of muscle, Proc R Soc Lond B Biol Sci 126 (1938), 136195.

[15] F.E. Zajac, Muscle and tendon: Properties, models, scaling, and application to biomechanics and motor control, Critical Reviews in Biomedical Engineering 17(4) (1989), 359-411.

[16] S. Annunziata, J. Paskarbeit and A. Schneider, Novel bioinspired control approaches to increase the stiffness variability in multi-muscle driven joints, Bioinspiraion \& Biomimetics 6 (2011), 045003.

[17] T.E. Milner, Dependence of elbow viscoelastic behavior on speed and loading in voluntary movements, Experimental Brain Research 93 (1993), 177-180.

[18] W.M. Murray, S.L. Delp and T.S. Buchanan, Variation of muscle moment arms with elbow and forearm position, Journal of Biomechanics 5 (1995), 513-525.

[19] M. Katayama and M. Kawato, Virtual trajectory and stiffness ellipse during multijoint arm movement predicted by neural inverse models, Biological Cybernetics 69 (1993), 353362.

[20] D. Bennett, J. Hollerbach, Y. Xu and I. Hunter, Time-varying stiffness of human elbow joint during cyclic voluntary movement, Experimental Brain Research 88 (1992), 433-442.

[21] F. Lacquaniti, F. Licata and J.F. Soechting, The mechanical behavior of the human forearm in response to transient perturbations, Biological Cybernetics 44 (1982), 35-46.

[22] W.M. Murray, T.S. Buchanan and S.L. Delp, The isometric functional capacity of muscles that cross the elbow, Journal of Biomechanics 33(8) (2000), 943-952.

[23] A.M. Smith, Does the cerebellum learn strategies for the optimal time-varying control of joint stiffness? Behavioral and Brain Sciences 19(3) (1996), 399-410.
[24] Z. Hasan, Optimized movement trajectories and joint stiffness in unperturbed, inertially loaded movements, Biological Cybernetics 53 (1986), 373-382.

[25] D.R. Humphrey and D.J. Reed, Separate cortical systems for control of joint movement and joint stiffness: Reciprocal activation and coactivation of antagonist muscles, Motor Control Mechanisms in Health and Disease 39(1) (1983), 347372.

[26] T.E. Milner and C. Cloutier, Damping of the wrist joint during voluntary movement, Experimental Brain Research, 122(3) (1998), 309-317.

[27] S.H. Stroeve, Impedance characteristics of a neuromusculoskeletal model of the human arm i. posture control, Biological Cybernetics (1999), 475-494.

[28] P. Giesl, D. Meisel, J. Scheurle and H. Wagner, Stability analysis of the elbow with a load, Journal of Theoretical Biology, 228(1) (2004), 115-125.

[29] A. Richardson, M. Tresch, E. Bizzi and J.-J. Slotine, Stability analysis of nonlinear muscle dynamics using contraction theory, In: Proceedings of the 27th Annual IEEE Engineering in Medicine and Biology Conference, Shangai, China, 2005, pp. 4986-4989.

[30] G. Raphael, G.A. Tsianos and G.E. Loeb, Spinal-like regulator facilitates control of a two-degree-of-freedom wrist, Journal of Neuroscience 30(28) (2010), 9431-9444.

[31] P. Giesl and H. Wagner, Lyapunov function and the basin of attraction for a single-joint muscle-skeletal model, Journal of Mathematical Biology 54 (2007), 453-464.

[32] A. Jacks, A. Prochazka and P.S.J. Trend, Instability in human forearm movements studied with feed-back-controlled electrical stimulation of muscles, Journal of Physiology 402 (1998), 443-461.

[33] E.R. Kandel, J.H. Schwartz and T.M. Jessell, Principles of Neural Science, E. Kandel, J. Schwartz and T.E. Jessell, Eds. McGraw-Hill, 4 edition, 2000, pp. 714-736.

[34] R. Shadmehr and S.P. Wise, The Computational Neurobiology of Reaching and Pointing: A Foundation for Motor Learning, MIT Press, Cambridge MA, 2005, pp. 108-118.

[35] J. McIntyre and E. Bizzi, Servo hypotheses for the biological control of movement, Journal of Motor Behavior 25(3) (1993), 93202.

[36] P.M. Mileusnic and G.E. Loeb, Mathematical models of proprioceptors. ii. structure and function of the golgi tendon organ, Journal of Neurophysiology 96 (2006), 17891802.

[37] M.L. Latash, Neurophysiological Basis of Movement. Second Edition, Human Kinetics, Urbana, IL, 2008, pp. 67-72.

[38] J. De Serres and T.E. Milner, Wrist muscle activation patterns and stiffness associated with stable and unstable mechanical loads, Experimental Brain Research 86(2) (1991), 451458.

[39] R.C. Frysinger, D. Bourbonnais, J.F. Kalaska and A.M. Smith, Cerebellar cortical activity during antagonist cocontraction and reciprocal inhibition of forearm muscles, Journal of Neurophysiology, 51(1) (1984), 32-49.

[40] B.P. Graham and S.J. Redman, Dynamic behaviour of a model of the muscle stretch reflex, Neural Networks 6 (1993), 947-962.

[41] C. Yang, G. Ganesh, S. Haddadin and S. Perusal, A. AlbuSchäffer and E. Burdet, Human-like adaptation of force and impedance in stable and unstable interactions, IEEE Transactions on Robotics 27 (2011), 918-930. 
[42] R.D. Woittiez, P.A. Huijing, H.B. Boom and R.H. Rozendal, A three-dimensional muscle model: A quantified relation between form and function of skeletal muscles, Journal of Morphology 182(1) (1984), 95-113.

[43] S. Annunziata, J. Paskarbeit and A. Schneider, Concurrent torque and stiffness control in an antagonistically actuated hinge joint is impaired by stiffness nodes, In: Proceedings of the 1st International Conference on Applied Bionics and Biomechanics (ICABB 2010), Venice, Italy, 2010. 

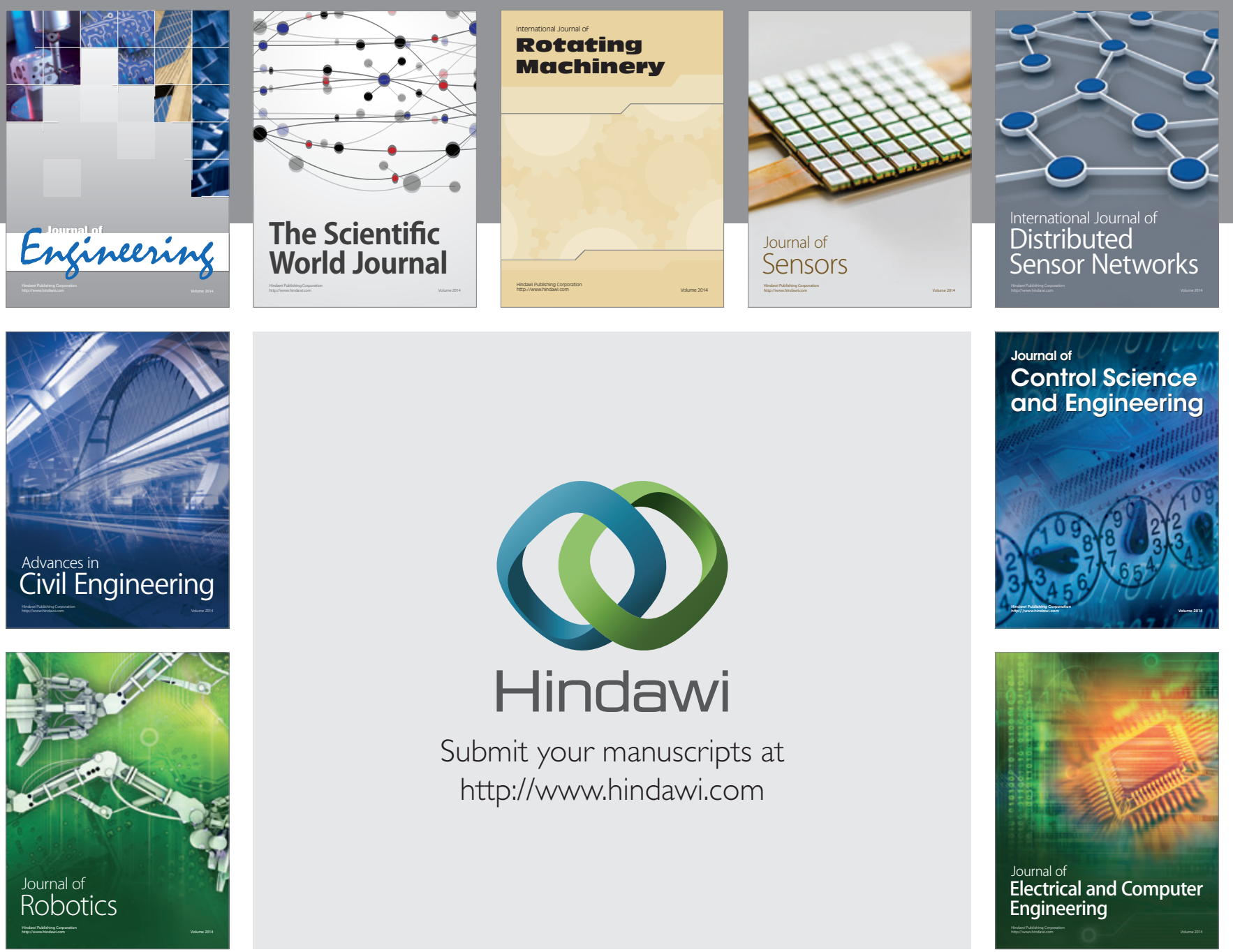

Submit your manuscripts at

http://www.hindawi.com
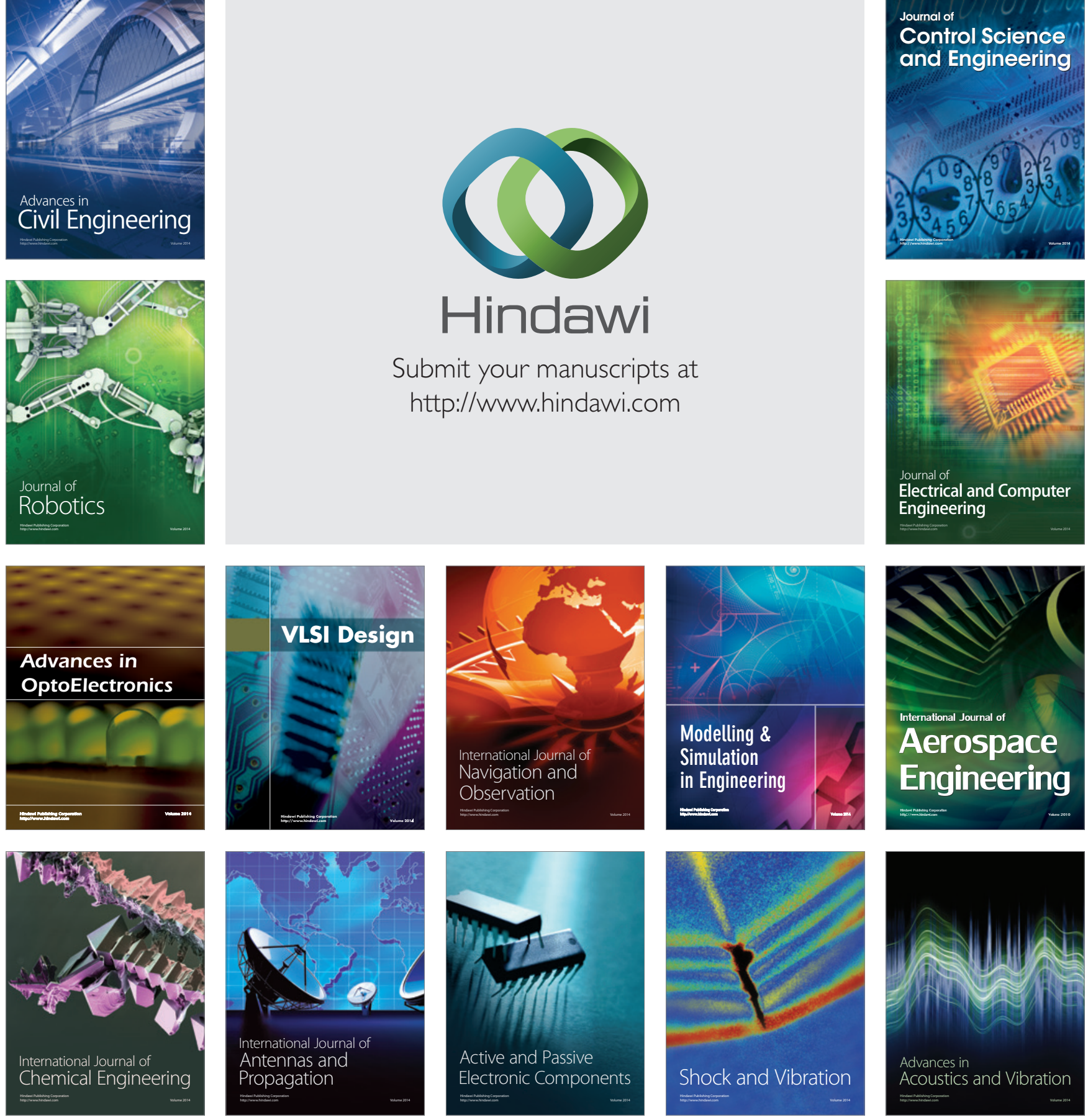